\title{
Vimentin-positive and Alpha-fetoprotein-elevated Nuclear Protein of the Testis Midline Carcinoma: A Case Report and Review of the Literature
}

\author{
Chihiro Murano, Shuhei Suzuki, Sho Nakamura, Hiroyuki Takeda, \\ Tadahisa Fukui and Takashi Yoshioka
}

\begin{abstract}
:
Nuclear protein of the testis (NUT) midline carcinoma (NMC) is a rare malignant tumor expressing NUT with $B R D 4 / 3$ rearrangements and is sometimes misdiagnosed as germinoma, especially in alpha-fetoprotein (AFP)-elevated cases. A 28-year-old man had a mediastinal tumor with multiple bone metastases and elevated AFP levels. Imaging/laboratory findings led to a pathological diagnosis of extragonadal germinoma. After unsuccessful treatment with etoposide-cisplatin, he was re-diagnosed with sarcoma due to vimentin-positive findings. He was treated with adriamycin-ifosfamide, which resulted in disease-control. A posthumous examination clarified the NUT rearrangement. Even in cases with characteristic findings, such as elevated AFP levels and vimentin positivity, NMC should be considered as a differential diagnosis. We note, however, that adriamycin-ifosfamide has some efficacy in such cases.
\end{abstract}

Key words: NUT midline carcinoma, AFP, vimentin, chemotherapy

(Intern Med 60: 3645-3649, 2021)

(DOI: 10.2169/internalmedicine.7019-21)

\section{Introduction}

Nuclear protein of the testis (NUT) midline carcinoma (NMC) is an uncommon and highly lethal neoplasm (1). The prognosis is extremely, poor, with a median survival of only 4.7 months in a previous study (2), and the median age at the onset is 21.9 years old (3). The hallmark of NMC is the genetic rearrangement of the NUT gene (on chromosome $15)$ with either $B R D 4[\mathrm{t}(15 ; 19)(\mathrm{q} 13.2 ; \mathrm{p} 13.1)]$ or $B R D 3$ (on chromosome 19), and in NMC cases, tumor cells show positive staining for NUT in immunochemical analyses (4-7).

In representative cases with NMC, malignancies frequently arise from the midline trunk or upper aerodigestive tract; such cases are associated with rapid progression, a poor response to cytotoxic agents and exceedingly poor differentiation $(8,9)$. They are sometimes initially diagnosed as other neoplasms, such as germ cell tumor (10), thymic cancer (11) or non-small-cell lung cancer (12). Indeed, a previous study reported that $50 \%$ of NMC patients were initially diagnosed with other malignancies (2). Furthermore, the diagnosis of midline tumors is challenging, and oncologists are forced to rely on tumor markers, the tumor location and morphologic findings in order to determine therapeutic strategies.

In young men with midline thorax tumors and elevated alpha-fetoprotein (AFP) levels, oncologists tend to administer a germ cell tumor regimen [bleomycin, etoposide and cisplatin (BEP)] (13-15). This is because the initial diagnosis is that of an extra-gonadal germ cell tumor, for which this treatment is expected to elicit a complete response. In cases where tumors have features of non-round-cell sarcomas with vimentin expression, oncologists tend to use an adriamycin-based regimen, as the initial diagnosis is that of non-round cell sarcoma (16), where at least a partial response is anticipated. Thus, clinicopathological features of elevated serum AFP levels or immunohistochemical vimentin expression are important not only for the making correct diagnosis but also for selecting the optimal chemotherapeutic regimen. 
a
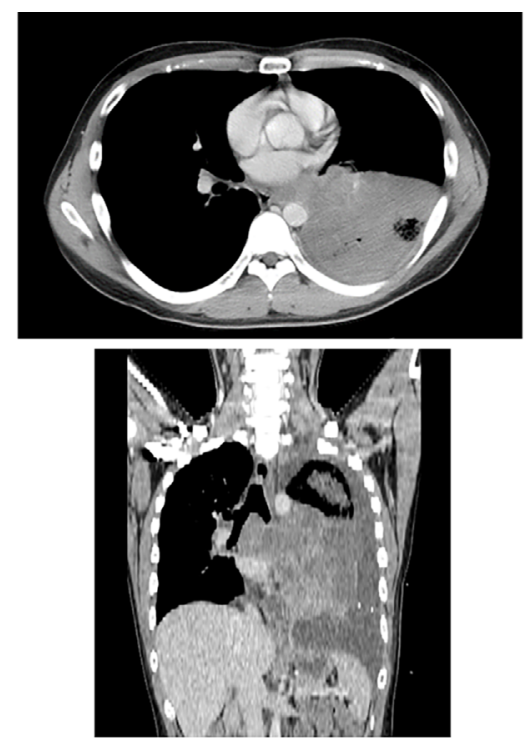

c

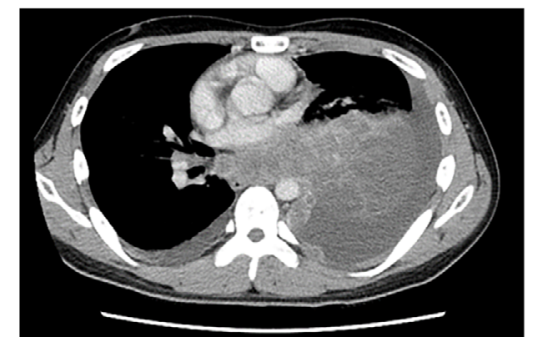

b
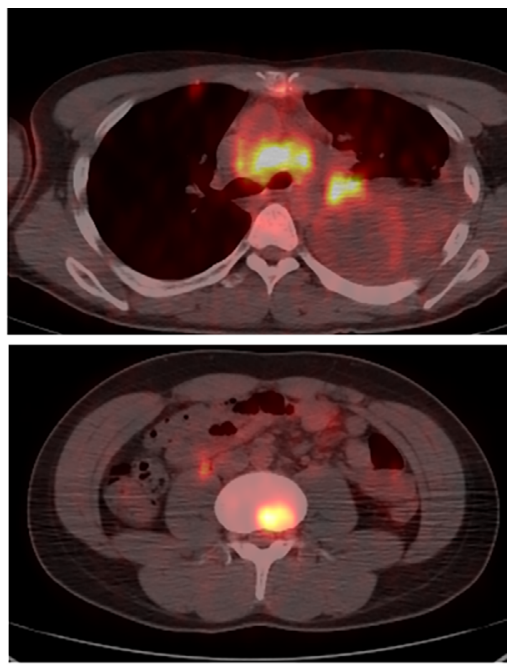

d

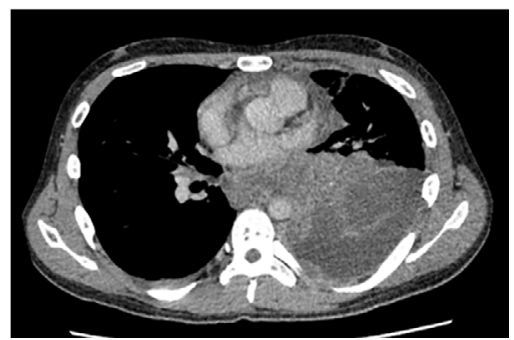

Figure 1. CT, PET/CT findings. a, b: Before etoposide and cisplatin treatment. The bulky tumor in the left thorax and left lung lower lobe collapse are shown. c: After etoposide and cisplatin treatment and before adriamycin and ifosfamide treatment. Note the increased pleural effusion and progressive dislocation of the heart and trachea. d: Shows virtually no tumor progression following adriamycin and ifosfamide treatment.

We herein report a young man with mediastinal tumor that was initially diagnosed as an extra-gonadal germ cell tumor due to elevated AFP levels (first-line BEP treatment response; poor). A subsequent diagnosis of non-round cell sarcoma was made due to the detection of vimentin expression (a moderate clinical response was elicited following second-line adriamycin and ifosfamide treatment). A final diagnosis of NMC was made posthumously upon a further examination.

\section{Case Report}

A 25-year-old Japanese man presented to us after experiencing a slight fever and cough for 3 weeks. His personal and family histories were unremarkable. He had smoked 10 cigarettes per day for 7 years and had a glass of beer a week. Whole-body computed tomography (CT) showed a large, 13-cm-diameter bulky tumor in the thorax with left lower lobe pulmonary collapse, indicating lung cancer (Fig. 1a). Since concomitant fluorodeoxyglucose-positron emission tomography $\left({ }^{18} \mathrm{~F}-\mathrm{FDG} \mathrm{PET}\right) / \mathrm{CT}$ showed bone metastases to multiple areas, including the vertebrae and bilateral femurs (Fig. 1b), an ultrasound-guided transbronchial mediastinum biopsy was performed.

Blood laboratory tests showed that AFP (74.6 ng/mL: nor- mal reference range $0.0-15.0 \mathrm{ng} / \mathrm{mL}$ ), lactate dehydrogenase (564 IU/L: normal reference range 120-234 IU/L) and Creactive protein $(5.97 \mathrm{mg} / \mathrm{dL}$ : normal reference range 0.0 $0.300 \mathrm{mg} / \mathrm{dL}$ ) levels were all elevated, while human chorionic gonadotropin and carcinoembryonic antigen levels were within the upper limit of the normal range. An analysis of the pathology led to the diagnosis of an extra-gonadal germ cell tumor.

Based on the patient's age, elevated AFP level, tumor location and pathological findings, a clinical diagnosis of an extra-gonadal germ cell tumor was made, and first-line treatment with germinoma-oriented chemotherapeutic agents was initiated. Specifically, the patient was treated with both etoposide and cisplatin, but without bleomycin in order to avoid pulmonary dysfunction.

However, contrary to expectations, the first-line treatment failed (Fig. 1c), and a secondary biopsy from a disseminated thorax tumor was performed immediately by laparoscopy in order to reevaluate the pathological status. After the rebiopsy and referral, the patient was transferred by ambulance to our hospital, provisionally in order to administer a second-line germinoma-oriented chemotherapy, such as paclitaxel, cisplatin and ifosfamide (so-called TIP regimen) (17). However, the second pathological report indicated a non-round-cell sarcoma, presenting with alveolar and 

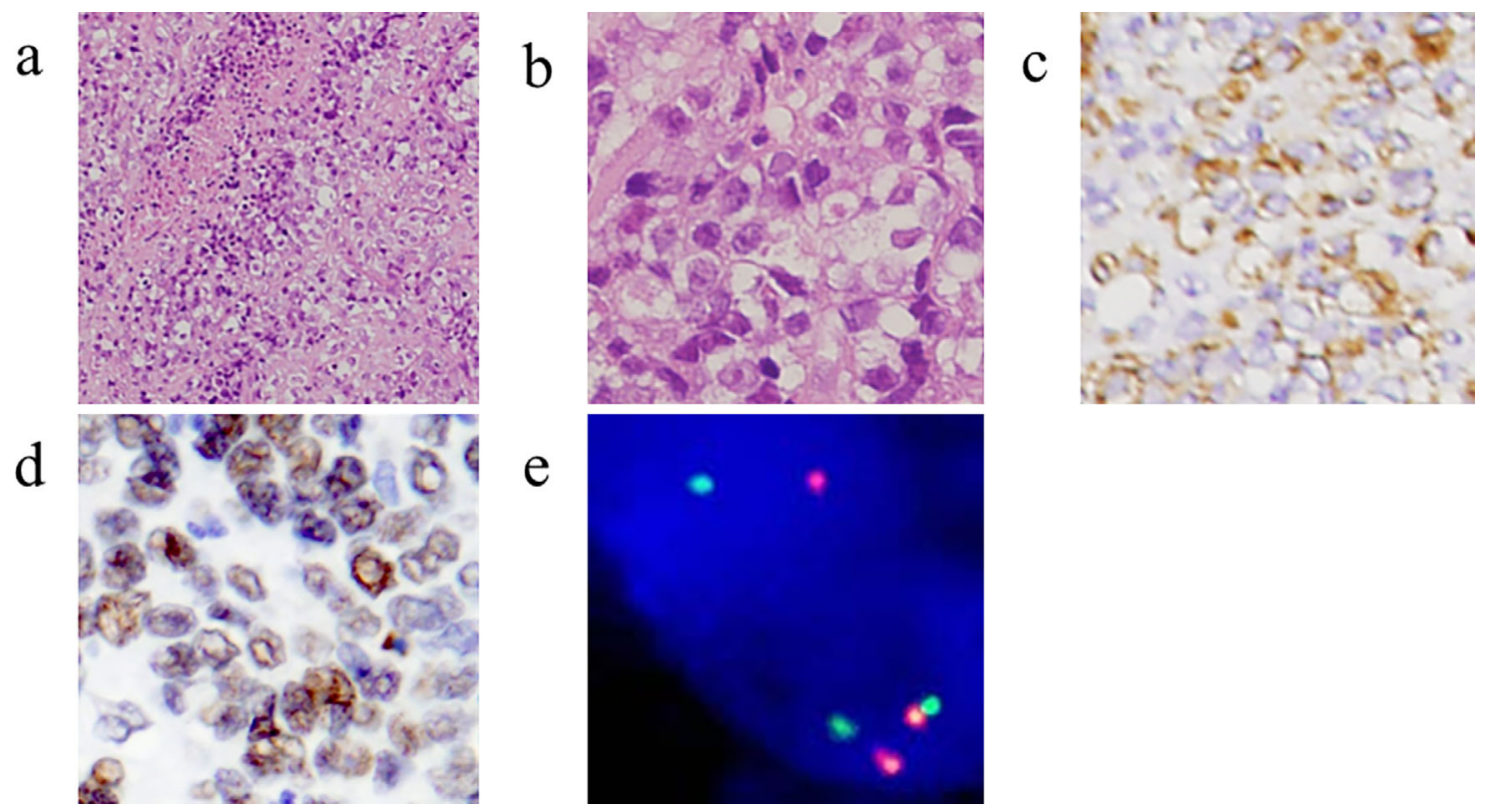

Figure 2. Pathological findings. a, b: Hematoxylin and Eosin staining (a: 40×magnification, b: 400×magnification). c, d: immunohistochemical staining [c: vimentin, d: NUT Family Member 1 (NUTM1)]. e: split signal of the NUTM1 gene on fluorescence in situ hybridization (82\%).

solid atypical cell growth with fibrous septum. The tumor was vimentin-positive (Fig. 2a-c) and focally positive for S100, melan-A, AE1/3, CD99 and EMA but negative for HMB45, KIT, CD30, AFP, hCG, CD20, CD79a, Desmin, CD34 and CD3.

Even though the patient had respiratory failure and needed oxygen, adriamycin and ifosfamide (sarcomaoriented regimen) treatment was initiated as a second-line chemotherapy in order to ameliorate the symptoms and prolong his life. After second-line chemotherapy, CT showed that the tumor had not progressed (Fig. 1d), and a blood laboratory test showed a gradual decrease in the AFP levels (nadir: $31.4 \mathrm{ng} / \mathrm{mL}$ ). Unfortunately, however, the effect of treatment did not last, and the overall survival duration from the second-line chemotherapy initiation was 73 days.

Advanced pathological examinations were subsequently performed, revealing the presence of an $82 \%$ split signal of NUT Family Member 1 (NUTM1) on fluorescence in situ hybridization and NUTM1 positivity on immunohistochemistry (Fig. 2d-e). The patient's next of kin provided written informed consent to prepare this report.

\section{Discussion}

Although a patient with NMC having both AFP elevation and vimentin expression has been previously reported (18), we believe this is the first report to show that adriamycinbased chemotherapy was effective to a degree in such a patient. It is difficult for doctors to diagnose mediastinum neoplasms due to their similarity to other malignancies (19). As such, biomarkers that can facilitate the proper treatment choice are urgently awaited, including tumor markers, histological information, immunohistochemical findings and clinical features. Hence, elevation of the serum AFP level (indicating an extra-gonadal germ cell tumor; AFP is a secretory protein, so it is not always positively-staining in tissues) or a vimentin-positive status (indicating a sarcoma) are critical indicators, and an examination of the genomic status is also warranted (12).

However, as in the present case, elevated AFP levels or vimentin positivity can prompt physicians to misdiagnose mediastinum neoplasm. There have been a number of reports of NMC cases in which AFP levels were elevated (Table 1) $(9,10,18,20)$ or in which the tumors were vimentinpositive (Table 2) $(18,21-24)$. Therefore, when noting elevated serum AFP levels or a vimentin-positive status, it is necessary to distinguish between NMC and other malignancies. Immunochemical staining for the NUT protein can be performed to facilitate the diagnosis of NMC (25).

We were unable to clarify the relationship between vimentin-positive staining and AFP elevation in this NUT case. Generally, poorly differentiated tumors might be more likely to be positive for vimentin than differentiated tumors (26), and AFP elevation is commonly observed in several types of tumors, indicating rapid progression $(27,28)$. Therefore, we speculate that vimentin-positive staining and AFP elevation were features indicating NMC's poor differentiation and aggressive progression.

Despite the small number of reported cases, patients with positively vimentin-stained NMC tend to follow a similar therapeutic clinical course, in that chemotherapy is initially effective but then fails, followed by a rapid, dismal progression $(18,23,24)$; this is precisely what we observed in the present case. Although the optimal chemotherapeutic regimen remains unclear, platinum-based $(1,2,10,18,20-23)$ or anthracycline-based $(1,2,29)$ regimens are the most 
Table 1. Case Reports of NUT Midline Carcinoma Presenting with Elevated Alpha Fetoprotein.

\begin{tabular}{|c|c|c|c|c|c|c|c|}
\hline Reference & Year & Sex & Age & Primary site & AFP & LDH & Treatment \\
\hline 18 & 2011 & M & 42 & Mediastinum & $659.9 \mathrm{ng} / \mathrm{mL}$ & Not known & $\begin{array}{l}\text { CDDP + IFO } \\
+ \text { VP-16 }\end{array}$ \\
\hline 21 & 2012 & $\mathrm{~F}$ & 19 & Lungs and ovary & $326 \mathrm{ng} / \mathrm{mL}$ & $1,982 \mathrm{U} / \mathrm{L}$ & $\begin{array}{c}\mathrm{CDDP}+\mathrm{IFO} \\
+\mathrm{VP}-16 \\
\mathrm{CBDCA}+\mathrm{PAC}\end{array}$ \\
\hline 22 & 2013 & M & 36 & Lung & $62 \mathrm{ng} / \mathrm{mL}$ & Not known & $\begin{array}{c}\text { CDDP + IFO } \\
+ \text { VP-16 } \\
\text { GSK525762 } \\
\text { (BET inhibitor) }\end{array}$ \\
\hline 9 & 2015 & $\mathrm{M}$ & 36 & Lung & $1,742.0 \mathrm{ng} / \mathrm{mL}$ & $741 \mathrm{U} / \mathrm{mL}$ & $\begin{array}{l}\text { CDDP + IFO } \\
+ \text { VP-16 }\end{array}$ \\
\hline 10 & 2016 & $\mathrm{M}$ & 28 & Mediastinum & $163.8 \mathrm{ng} / \mathrm{mL}$ & $667 \mathrm{IU} / \mathrm{L}$ & $\begin{array}{c}\mathrm{BLM}+\mathrm{CDDP} \\
+\mathrm{VP}-16 \\
\mathrm{DXR} \\
\text { DOC }\end{array}$ \\
\hline 20 & 2017 & M & 22 & Mediastinum & $765 \mathrm{ng} / \mathrm{mL}$ & $14,468 \mathrm{U} / \mathrm{mL}$ & $\mathrm{CDDP}+\mathrm{VP}-16$ \\
\hline Present report & 2020 & $\mathrm{M}$ & 25 & Lung & $74.6 \mathrm{ng} / \mathrm{mL}$ & $564 \mathrm{IU} / \mathrm{L}$ & $\begin{array}{c}\mathrm{CDDP}+\mathrm{VP}-16 \\
\mathrm{DXR}+\mathrm{IFO}\end{array}$ \\
\hline
\end{tabular}

Table 2. Case Reports of Vimentin-positive NUT Midline Carcinoma.

\begin{tabular}{|c|c|c|c|c|c|c|}
\hline & Year & Sex & Age & Primary site & Vimentin & Treatment \\
\hline 18 & 2011 & $\mathrm{M}$ & 42 & Mediastinum & Positive & $\begin{array}{c}\text { CDDP + IFO } \\
+ \text { VP-16 }\end{array}$ \\
\hline 23 & 2011 & $\mathrm{~F}$ & 54 & Nasal dorsum & Positive & $\begin{array}{c}\text { CDDP } \\
\text { VP-16 + IFO }\end{array}$ \\
\hline 24 & 2014 & $\mathrm{~F}$ & 18 & Nasal cavity & Positive & Not known \\
\hline Present report & 2020 & M & 25 & Lung & Positive & $\begin{array}{c}\text { CDDP + VP-16 } \\
\text { DXR + IFO }\end{array}$ \\
\hline
\end{tabular}

commonly used to date. However, the treatment outcome remains dismal, prompting intense research aimed at improved clinical management. More effective chemotherapeutic agents are also needed, along with new molecular-targeted agents: there is particular interest in epigenetic therapies using histone deacetylase or bromodomain and extra-terminal (BET) inhibitors (30). Furthermore, novel treatment modalities such as targeting microRNAs (miRs) are showing promise. In the case of NMC, miR-3140 has recently been reported as a potential therapeutic target (31). More basic and clinical research is clearly needed in order to improve the NUT treatment outcome.

The authors state that they have no Conflict of Interest (COI).

\section{Acknowledgement}

We would like to thank NAI for English language editing, and Tadashi Hasegawa, Department of Surgical Pathology, Sapporo medical University School of Medicine for pathological findings/ photos.

\section{References}

1. Bauer DE, Mitchell CM, Strait KM, et al. Clinicopathologic features and long-term outcomes of NUT midline carcinoma. Clin Cancer Res 18: 5773-5779, 2012.
2. Lemelle L, Pierron G, Freneaux P, et al. NUT carcinoma in children and adults: a multicenter retrospective study. Pediatr Blood Cancer 64: 10.1002/pbc.26693, 2017.

3. Chau NG, Hurwitz S, Mitchell CM, et al. Intensive treatment and survival outcomes in NUT midline carcinoma of the head and neck. Cancer 122: 3632-3640, 2016.

4. French CA, Rahman S, Walsh EM, et al. NSD3-NUT fusion oncoprotein in NUT midline carcinoma: implications for a novel oncogenic mechanism. Cancer Discov 4: 928-941, 2014.

5. Alekseyenko AA, Walsh EM, Wang X, et al. The oncogenic BRD 4-NUT chromatin regulator drives aberrant transcription within large topological domains. Genes Dev 29: 1507-1523, 2015.

6. French CA, Miyoshi I, Kubonishi I, Grier HE, Perez-Atayde AR, Fletcher JA. BRD4-NUT fusion oncogene: a novel mechanism in aggressive carcinoma. Cancer Res 63: 304-307, 2003.

7. French CA, Ramirez CL, Kolmakova J, et al. BRD-NUT oncoproteins: a family of closely related nuclear proteins that block epithelial differentiation and maintain the growth of carcinoma cells. Oncogene 27: 2237-2242, 2008.

8. French CA. NUT midline carcinoma. Cancer Genet Cytogenet 203: 16-20, 2010.

9. Raza A, Cao H, Conrad R, et al. Nuclear protein in testis midline carcinoma with unusual elevation of alpha-fetoprotein and synaptophysin positivity: a case report and review of the literature. Expert Rev Anticancer Ther 15: 1199-1213, 2015.

10. Harada Y, Koyama T, Takeuchi K, Shoji K, Hoshi K, Oyama Y. NUT midline carcinoma mimicking a germ cell tumor: a case report. BMC Cancer 16: 895, 2016.

11. Gokmen-Polar Y, Kesler K, Loehrer PJ Sr, Badve S Sr. NUT 
midline carcinoma masquerading as a thymic carcinoma. J Clin Oncol 34: e126-e129, 2016.

12. Baras AS, Naidoo J, Hann CL, Illei PB, Reninger CW 3rd, Lauring J. Rediagnosis of lung cancer as NUT midline carcinoma based on clues from tumor genomic profiling. J Natl Compr Cancer Netw 16: 467-472, 2018.

13. Williams SD, Birch R, Einhorn LH, Irwin L, Greco FA, Loehrer PJ. Treatment of disseminated germ-cell tumors with cisplatin, bleomycin, and either vinblastine or etoposide. New Engl J Med 316: 1435-1440, 1987.

14. Hainsworth JD, Williams SD, Einhorn LH, Birch R, Greco FA Successful treatment of resistant germinal neoplasms with VP-16 and cisplatin: results of a Southeastern Cancer Study Group trial. J Clin Oncol 3: 666-671, 1985.

15. Greco FA, Vaughn WK, Hainsworth JD. Advanced poorly differentiated carcinoma of unknown primary site: recognition of a treatable syndrome. Ann Intern Med 104: 547-553, 1986.

16. Bramwell VH, Anderson D, Charette ML. Doxorubicin-based chemotherapy for the palliative treatment of adult patients with locally advanced or metastatic soft tissue sarcoma. Cochrane Database Syst Rev Cd003293, 2003.

17. Kondagunta GV, Bacik J, Donadio A, et al. Combination of paclitaxel, ifosfamide, and cisplatin is an effective second-line therapy for patients with relapsed testicular germ cell tumors. J Clin Oncol 23: 6549-6555, 2005

18. Zhu B, Laskin W, Chen Y, et al. NUT midline carcinoma: a neoplasm with diagnostic challenges in cytology. Cytopathology 22: 414-417, 2011

19. Clamon GH. Management of primary mediastinal seminoma. Chest 83: 263-267, 1983.

20. D’Ambrosio L, Palesandro E, Moretti M, et al. Alpha-fetoprotein elevation in NUT midline carcinoma: a case report. BMC Cancer 17: 266, 2017.

21. Ball A, Bromley A, Glaze S, French CA, Ghatage P, Kobel M. A rare case of NUT midline carcinoma. Gynecol Oncol Case Rep 3 $1-3,2012$.

22. Parikh SA, French CA, Costello BA, et al. NUT midline carcinoma: an aggressive intrathoracic neoplasm. J Thorac Oncol 8:
1335-1338, 2013.

23. Davis BN, Karabakhtsian RG, Pettigrew AL, Arnold SM, French CA, Brill YM. Nuclear protein in testis midline carcinomas: a lethal and underrecognized entity. Arch Pathol Lab Med 135: 14941498, 2011.

24. Suzuki S, Kurabe N, Minato H, et al. A rare Japanese case with a NUT midline carcinoma in the nasal cavity: a case report with immunohistochemical and genetic analyses. Pathol Res Pract 210: 383-388, 2014.

25. Haack H, Johnson LA, Fry CJ, et al. Diagnosis of NUT midline carcinoma using a NUT-specific monoclonal antibody. Am J Surg Pathol 33: 984-991, 2009.

26. Upton MP, Hirohashi S, Tome Y, Miyazawa N, Suemasu K, Shimosato Y. Expression of vimentin in surgically resected adenocarcinomas and large cell carcinomas of lung. Am J Surg Pathol 10: $560-567,1986$

27. McIntire KR, Waldmann TA, Moertel CG, Go VL. Serum alphafetoprotein in patients with neoplasms of the gastrointestinal tract. Cancer Res 35: 991-996, 1975.

28. Liu X, Cheng Y, Sheng W, et al. Clinicopathologic features and prognostic factors in alpha-fetoprotein-producing gastric cancers: analysis of 104 cases. J Surg Oncol 102: 249-255, 2010.

29. Watanabe S, Hirano S, Mine S, et al. A case of endobronchial NUT midline carcinoma with intraluminal growth. Anticancer Res 35: 1607-1612, 2015.

30. Bhadury J, Nilsson LM, Muralidharan SV, et al. BET and HDAC inhibitors induce similar genes and biological effects and synergize to kill in Myc-induced murine lymphoma. Proc Natl Acad Sci USA 111: E2721-E2730, 2014.

31. Tonouchi E, Gen Y, Muramatsu T, et al. miR-3140 suppresses tumor cell growth by targeting BRD4 via its coding sequence and downregulates the BRD4-NUT fusion oncoprotein. Sci Rep 8: 4482, 2018.

The Internal Medicine is an Open Access journal distributed under the Creative Commons Attribution-NonCommercial-NoDerivatives 4.0 International License. To view the details of this license, please visit (https://creativecommons.org/licenses/ by-nc-nd/4.0/).

(C) 2021 The Japanese Society of Internal Medicine

Intern Med 60: 3645-3649, 2021 